\title{
Spectral analyses of trans- and cis-DOCO transients via comb spectroscopy
}

Thinh Q. Bui ${ }^{1}$, P. Bryan Changala ${ }^{1}$, Bryce J. Bjork ${ }^{2}$, Qi Yu ${ }^{3}$, Yimin Wang ${ }^{4}$, John F. Stanton $^{5}$, Joel Bowman ${ }^{3}$, Jun $\mathrm{Ye}^{1}$

${ }^{1}$ JILA, National Institute of Standards and Technology, and Department of Physics, University of Colorado, Boulder, CO 80309, USA

${ }^{2}$ Honeywell International, 303 Technology Ct., Broomfield, Colorado, 80021, USA

${ }^{3}$ Cherry L. Emerson Center for Scientific Computation and Department of Chemistry, Emory University, Atlanta, Georgia 30322, United States

${ }^{4} 2604$ Kings Lake Court NE, Atlanta, Georgia 30345, USA

${ }^{5}$ Department of Chemistry, University of Florida, Gainesville, Florida 32611, USA

\section{Abstract}

We use time-resolved direct frequency comb spectroscopy in the mid-infrared to obtain high-resolution rovibrational spectra of products produced from the OD+CO reaction. In this work, we present spectral analyses for isotopologues of the transient DOCO radicals from this reaction in the OD stretch region. The analyses were performed with aid of two different theoretical approaches based on both perturbation theory and variational calculations used for prediction of rovibrational spectra of polyatomic molecules. We discuss the advantages and challenges of our current approach for studying spectroscopy and dynamics of transient molecules.

Keywords: frequency comb, DOCO, infrared spectroscopy, CFOUR, MULTIMODE 


\section{Introduction}

The use of time-resolved spectroscopy for the study of elementary reaction processes, a key driver in the fundamental understanding of chemical reaction mechanisms and molecular dynamics [1], has experienced revolutionary transformation beginning from Norrish and Porter's seminal flash photolysis experiment to ultrafast "femtochemistry" by Ahmed Zewail [2]. The development of ultrafast lasers served as a cornerstone for this transition. Taking a different path, high resolution spectroscopy and precision measurement have motivated the development of stable lasers and frequencydomain approaches. The great merge of these two scientific paths led to the eventual development of the optical frequency comb [3]. The frequency comb possesses broad spectral bandwidth and high spectral resolution in the frequency domain, making it a suitable light source for high-resolution spectroscopy in what has been termed "direct frequency comb spectroscopy" (DFCS) [4]. The versatility of DFCS has more recently been extended to studies of high resolution spectroscopy of large molecules $[5,6]$ and chemical kinetics [7-10]. Continuing efforts are focused towards construction of high power frequency comb sources that cover 5 to $10 \mu \mathrm{m}$ for future advances in high resolution molecular spectroscopy and dynamics [11].

In Ref. [7] we reported the use of cavity-enhanced direct frequency comb spectroscopy to determine the real-time kinetics of the OD+CO reaction, which is important in atmospheric and combustion chemistry [12]. When combined with a dispersive spectrometer, this technique achieves the time resolution necessary for monitoring real-time formation and decay of the reaction intermediate (DOCO) and product $\left(\mathrm{CO}_{2}\right)$ from the $\mathrm{OD}+\mathrm{CO}$ reaction. Here, we provide a detailed presentation on the spectral analyses of the reaction products, specifically the intermediate transients of trans- and cis-DOCO, based on high resolution spectroscopy data in the OD stretch band 
region $(\lambda \sim 3.7$ to $4.2 \mu \mathrm{m})$. These spectroscopic investigations form an important part of our understanding of the structure and dynamics of these transient species [13].

High-resolution spectra of $\mathrm{H}(\mathrm{D}) \mathrm{OCO}$ intermediates motivate the development of a more accurate $\mathrm{OH}(\mathrm{D})+\mathrm{CO}$ global potential energy surface (PES), especially in the low energy regions probed by observation of vibrational fundamentals in the infrared wavelengths $[14,15,16]$. Relying on ab initio PES, recent theoretical work has focused on the dissociation dynamics of $\mathrm{H}(\mathrm{D}) \mathrm{OCO}$ isomers to $\mathrm{OH}(\mathrm{D})+\mathrm{CO}$ or $\mathrm{H}(\mathrm{D})+\mathrm{CO}_{2}$ products $[15,17]$. Gas phase infrared spectroscopy of trans-H(D)OCO have now been reported for the $v_{1} \mathrm{O}-\mathrm{H}(\mathrm{D})$ stretch [18-20] and $v_{2} \mathrm{C}=\mathrm{O}$ stretch [21] vibrational fundamental modes. Extensive microwave and millimeter-wave studies for the vibrational ground state have also been reported for both trans and cis isomers [22-24]. The spectroscopic data reported in this work thus provides important information for a more comprehensive understanding of the structure of DOCO isomers.

\section{Methods}

\section{Transient DOCO production}

Detailed descriptions of the DOCO-forming reaction processes have been described in our previous work [9]; only a brief review will be given here. First, $\mathrm{O}_{3}$ gas is photo-dissociated in a room temperature reaction cell (continuous gas flow) by a 266 nm pulse (10 ns, $35 \mathrm{~mJ} /$ pulse) to produce $\mathrm{O}\left({ }^{1} \mathrm{D}\right)$ and $\mathrm{O}_{2}$. In the presence of $\mathrm{D}_{2}$, the reaction of $\mathrm{O}\left({ }^{1} \mathrm{D}\right)+\mathrm{D}_{2}$ produces $\mathrm{OD}$ radicals. $\mathrm{CO}$ is then added to initiate the $\mathrm{OD}+\mathrm{CO}$ reaction, which produces reactive intermediates cis- and trans-DOCO and product $\mathrm{CO}_{2}$. This work will focus on the high-resolution spectroscopy and supporting rovibrational calculations for the DOCO intermediates (trans-DO ${ }^{12} \mathrm{CO}$, trans $-\mathrm{DO}^{13} \mathrm{CO}$, cis-DO ${ }^{12} \mathrm{CO}$, and cis$\left.\mathrm{DO}^{13} \mathrm{CO}\right)$ in the $\mathrm{OD}(\mathrm{v}=1)$ stretch region. 


\section{Time-resolved frequency comb spectroscopy}

The mid-IR frequency comb light is produced from a tunable $(\lambda \sim 3$ to $5 \mu \mathrm{m})$ optical parametric oscillator (OPO) synchronously pumped with an $10 \mathrm{~W}$ ytterbium fiber $\operatorname{comb}(\lambda \sim 1.06 \mu \mathrm{m})$ [25]. In this work, the OPO wavelength is tuned from $3.6 \mu \mathrm{m}$ to 4.3 $\mu \mathrm{m}\left(2300\right.$ to $2800 \mathrm{~cm}^{-1}$, average power $\sim 200$ to $\left.500 \mathrm{~mW}\right)$. The repetition rate $\left(f_{\text {rep }}\right)$ of the comb is $~ 136.7 \mathrm{MHz}$.

Light from the OPO is sent into an optical cavity (which also served as the reaction cell) enclosed by two high reflectivity mirrors for cavity-enhanced absorption spectroscopy. The measured finesse of the cavity is shown in Fig. 1A. The length of the cavity is approximately $54.9 \mathrm{~cm}$ resulting in a cavity free spectral range (FSR) of $\sim 273$ $\mathrm{MHz}$, or $2 \times f_{\text {rep. }}$. Therefore, every other comb mode is coupled into the cavity. In this experiment, photo-dissociation of $\mathrm{O}_{3}$ by the $\mathrm{Nd}$ : $\mathrm{YAG}$ laser causes a transient increase in the gas pressure, which changes the effective optical path length. In the case of a tight comb-cavity locking method like the PDH lock, this sudden disturbance results in lock instability and/or introduces cavity transmission noise (frequency-to-amplitude noise conversion). Therefore, to maintain cavity transmission, the swept cavity lock method [26] was used rather than the PDH lock method. Here, the comb $f_{\text {rep }}$ is modulated at 50 $\mathrm{kHz}$, and the cavity transmission signal is demodulated and fed back to the cavity piezo to keep the cavity FSR locked to the $f_{\text {rep }}$ of the comb laser. At the expense of lowering the laser-cavity coupling duty cycle (decreased cavity transmission), the swept cavity lock technique has the advantage of being less sensitive to the photolysis process.

The transmitted comb spectrum is dispersed by a spectrometer that comprises a combination of a virtually-imaged phased array (VIPA) etalon [27] and a reflective diffraction grating. Since the cavity-filtered comb mode spacing (273 MHz) cannot be 
resolved by the VIPA etalon, the spectrometer sets the resolution ( $900 \mathrm{MHz})$ rather than the linewidth of the comb mode $(\sim 50 \mathrm{kHz})$. Output from the VIPA etalon (vertical dispersion) is cross-dispersed with a grating (horizontal dispersion), and imaged on an InSb camera. The integration time of the camera sets the time resolution ( $\left.t_{\text {int }} \geq 10 \mu \mathrm{s}\right)$. This configuration allows for simultaneous measurement of approximately $65 \mathrm{~cm}^{-1}$ of the comb spectral width at a VIPA limited resolution of $\sim 900 \mathrm{MHz}\left(\sim 0.03 \mathrm{~cm}^{-1}\right)$. This corresponds to more than 2000 spectrally resolved elements that are acquired simultaneously within $10 \mu \mathrm{s}$. The experiments are conducted at 100 Torr and room temperature, which means that the combined Doppler and pressure broadened lineshape exceeds the VIPA limited resolution. Thus, the experimental conditions for studying the $\mathrm{OD}+\mathrm{CO}$ reaction ultimately determine the spectral resolution, not the frequency comb or spectrometer.

The dispersive spectrometer provides the necessary time resolution to observe the short-lived (100 $\mu \mathrm{s})$ DOCO intermediates via a pump-comb probe experiment with the Nd:YAG photolysis (pump) laser. The integration of the cavity transmitted comb light on the InSb camera is synchronized with the photolysis pulse. To obtain a direct absorption signal, rapid successive acquisitions of the reference ' $R$ ' (pre-photolysis) and signal ' $S$ ' (post-photolysis) camera images are recorded. The experimentally chosen temporal separation between the $\mathrm{R}$ and $\mathrm{S}$ defines the reaction kinetics time. The absorbance is determined from

$$
A=-\ln \left(\frac{S-B}{R-B}\right) .
$$

eq. 1

In eq. 1, ' $B$ ' refers to the background camera image with the IR beam blocked by a mechanical shutter, which is measured $4 \mathrm{~ms}$ before the $\mathrm{R}$ image. Fast subtraction of the 
B image from both the $\mathrm{R}$ and $\mathrm{S}$ mitigates additional noise caused by the temperaturedependent dark current offset drifts of the InSb camera.

The duty cycle of the experiment is limited by the $10 \mathrm{~Hz}$ repetition rate of the Nd:YAG laser. The $100 \mathrm{~ms}$ separation between photolysis pulses provides more than sufficient time for gas pump out (residence time is $\sim 20 \mathrm{~ms}$ ). Due to latency in the acquisition software, the actual acquisition repetition rate is approximately $3 \mathrm{~Hz}$. The single shot absorption sensitivity is estimated by the noise-equivalent absorption (NEA) per spectral element (to normalize the comb bandwidth), which is given by

$$
\mathrm{NEA}=\sigma_{\mathrm{A}} \frac{\pi}{F L_{p}} \sqrt{\frac{T}{M}} .
$$

Here, $\sigma_{\mathrm{A}}$ is the standard deviation in the single shot absorbance calculated by eq. $1, F$ is the cavity finesse, $L_{\mathrm{p}}$ is the photolysis pathlength, $T$ is the total period for the measurement of $A$, and $M$ is the number of resolvable spectral elements per camera image. At peak finesse, NEA is $2 \times 10^{-10} \mathrm{~cm}^{-1} \mathrm{~Hz}^{-1 / 2}$, which is a factor of five better than the previous timeresolved frequency comb experiment with reported a NEA of $1.1 \times 10^{-9} \mathrm{~cm}^{-1} \mathrm{~Hz}^{-1 / 2}$ [10]. The improvement can be attributed to higher cavity finesse in the current apparatus.

To further enhance the absorption sensitivity, many single shot spectra are averaged. Fig. 1B shows the Allan deviation of the absorbance at two different spectral baseline points as a function of averaging time $\tau$. Here, the baseline noise averages down as $\tau^{-1 / 2}$ even after 30 minutes of averaging (at a $3 \mathrm{~Hz}$ acquisition rate). This observation reveals the additional noise reduction advantage of our $10 \times$ faster differential measurement compared to a previous study [5].

\section{Rovibrational calculations}
A. MULTIMODE 
We have performed vibrational self-consistent field/virtual state configuration interaction (VSCF/VCI) calculations, as implemented in the code MULTIMODE (MM) [28]. The exact Watson Hamiltonian is used in the representation of mass-scaled normal modes. For all the cases, we use the 6-mode representation of the potential (no approximation made). The two different potential energy surfaces used are developed by Chen et al. [29] and Wang et al. [16] The former PES is a global surface starting from the $\mathrm{OH}+\mathrm{CO}$ asymptote to the $\mathrm{H}+\mathrm{CO}_{2}$ product, while the latter is centered around the minima of the trans- and cis-HOCO isomers. For each normal mode, twenty-two Gauss quadrature points are selected in generating a set of harmonic basis functions. In VCI calculations, the sum of mode excitations of all 6 normal modes are 14, 14, 13, 13, 11,10 for 1-mode to 6-mode excitations. The final size of VCI matrix is 20877.

\section{B. VPT2}

Separately, we also have performed second-order vibrational perturbation theory (VPT2) [30] calculations for both the ${ }^{12} \mathrm{C}$ and ${ }^{13} \mathrm{C}$ isotopologues of the cis and trans isomers. Standard semi-diagonal quartic force fields with respect to the rectilinear normal coordinates are calculated at the frozen-core $\operatorname{CCSD}(\mathrm{T})$ level of theory with the ANO1 basis set [31] using the CFOUR package [32]. The VPT2 predictions include both anharmonic vibrational frequencies and vibrational corrections to the rotational constants.

\section{Results and discussions}

The calculated DOCO vibrational frequencies obtained using MULTIMODE and VPT2 are compiled in Tables 1 and 2, respectively. For trans-DOCO, the ${ }^{13} \mathrm{C}$ substitution is not anticipated to significantly shift the origin of the OD stretch band relative to ${ }^{12} \mathrm{C}$, as corroborated by the nearly identical computed values for the two carbon isotopologues. For cis-DOCO, the predicted values for both the ${ }^{12} \mathrm{C}$ and ${ }^{13} \mathrm{C}$ isotopologues provide 
guidance for our search for the cis-DOCO radical in the OD stretch vibrational band. For this purpose, MULTIMODE using two different PES and VPT2 all provided good agreement for the OD stretch frequency within $\sim 10 \mathrm{~cm}^{-1}$.

The absorption spectrum of each major species produced from the $\mathrm{OD}+\mathrm{CO}$ reaction is shown in Fig. 2. The large bandwidth of the high reflectivity mirrors spans a measurement range of $2380 \mathrm{~cm}^{-1}$ to $2760 \mathrm{~cm}^{-1}$ (3.6 $\mu \mathrm{m}$ to $\left.4.2 \mu \mathrm{m}\right)$, which allows us to measure $\mathrm{CO}_{2}, \mathrm{DO}_{2}, \mathrm{D}_{2} \mathrm{O}, \mathrm{OD}$, cis- and trans-DOCO. The simulated $\mathrm{OD}$ and $\mathrm{D}_{2} \mathrm{O}$ line positions are obtained from Abrams et al. [33] and Toth et al. [34], respectively. The $\mathrm{DO}_{2}$ spectrum is simulated from measured rovibrational constants from Lubic et al. [35] All spectra are simulated at $\mathrm{T}=295 \mathrm{~K}$, including that of the DOCO isomers, which, despite being produced with significant chemical activation from the $\mathrm{OD}+\mathrm{CO}$ reaction, are rapidly thermalized to room temperature by high background concentrations of $\mathrm{N}_{2}$ and CO.

\section{I. trans-DOCO}

trans-DOCO is a planar, near-prolate asymmetric top. The ratio of the a-type to b-type integrated band intensities for its OD stretch fundametnal is estimated to be $\left|\mu_{\mathrm{a}} / \mu_{\mathrm{b}}\right|^{2}$ $\sim 3$ based on jet-cooled spectra of trans-HOCO [20]. Despite the similar band intensities, previous room temperature vibrational spectra in the $\mathrm{OH}(\mathrm{D})$ stretch region of trans$\mathrm{H}(\mathrm{D}) \mathrm{O}^{12} \mathrm{CO}$ are dominated by a-type transitions with no apparent signatures of b-type transitions $[18,19]$, which is consistent with our own measurements. For the trans$\mathrm{DO}^{12} \mathrm{CO}$ isotopologue, both of ground state [23,24] and excited $\mathrm{OD}(\mathrm{v}=1)$ stretch [18] rovibrational constants have been previously reported, so we will not discuss that here.

We report infrared spectroscopy of the trans-DO ${ }^{13} \mathrm{CO}$ isotopologue, for which no previous reports have been made. Figure 3 show the experimental and fitted (inverted) spectra for trans-DO ${ }^{13} \mathrm{CO}$. The fits utilize parameters for the Watson A-reduced effective 
Hamiltonian ( $\mathrm{I}^{\mathrm{r}}$ representation) and are performed using PGOPHER [36]. Since there is no previous measurement of this vibrational band, the previously described VPT2 calculations provided initial guesses for both the vibrational band origin and rotational constants (A, B, C).

The rotational energies of a near-prolate asymmetric top increase approximately as $(\mathrm{A}-(\mathrm{B}+\mathrm{C}) / 2) \mathrm{K}_{\mathrm{a}}^{2}$ [the $\mathrm{K}_{\mathrm{a}}$ quantum number is the projection of the rotational angular momentum along the principal a-axis]. The propensity rule for a-type transitions is $\Delta \mathrm{K}_{\mathrm{a}}=0$, while for b-type transitions it is $\Delta \mathrm{K}_{\mathrm{a}}= \pm 1$. The rotational constant A, which largely determines the spacing between different $\mathrm{K}_{\mathrm{a}}$ stacks, is poorly constrained in an atype spectrum because of the $\Delta \mathrm{K}_{\mathrm{a}}=0$ propensity rule. By only observing a-type transitions in this experiment, the strong correlation in the fitted values of $\mathrm{A}, \mathrm{B}$, and $\mathrm{C}$ precludes their accurate individual determination. Thus the values for the rotational constant $\mathrm{A}$ for the ground $\left(\mathrm{A}_{0}\right)$ and vibrationally excited state $\left(\mathrm{A}_{\mathrm{v}}\right)$ are determined by correcting the experimental ${ }^{12} \mathrm{C}$ values with the calculated VPT2 isotopic shift and fixed in the fit. The quartic centrifugal distortion terms are fixed to the experimental trans- $\mathrm{DO}^{12} \mathrm{CO}$ values reported by Petty and Moore [18] for both carbon isotopologues. The instrument and pressure-broadened transitions (linewidth $\sim 0.03 \mathrm{~cm}^{-1}$ ) for the DOCO isomers cannot resolve the asymmetry doubling (for levels $K_{a} \neq 0$ ), which constrains the difference in $B$ and $\mathrm{C}$. Therefore, only the average value of $(\mathrm{B}+\mathrm{C}) / 2$ for the ground $\left(\mathrm{B}_{0}\right.$ and $\left.\mathrm{C}_{0}\right)$ and vibrationally excited states $\left(\mathrm{B}_{\mathrm{v}}\right.$ and $\left.\mathrm{C}_{\mathrm{v}}\right)$ are fitted for trans- $\mathrm{DO}^{13} \mathrm{CO}$, along with the $\mathrm{v}=1$ band origin.

The fitted trans-DO ${ }^{13} \mathrm{CO}$ rovibrational constants are compiled in Table 3. The standard deviation of the fit is $\sim 0.013 \mathrm{~cm}^{-1}$, which is well below the uncertainty of $\sim 0.1$ $\mathrm{cm}^{-1}$ for the experimental transition energies. The observed agreement between the measured and predicted spectra demonstrates that only a few free parameters (the average 
of the $\mathrm{B}$ and $\mathrm{C}$ rotational constants and the band origin) are required to reproduce the pressure-broadened, room temperature spectrum of trans $-\mathrm{DO}^{13} \mathrm{CO}$ to within experimental uncertainty.

\section{II. cis-DOCO}

Prior to our work, there have been no previous reports of the gas phase vibrational spectrum of the cis-H(D)OCO isomer. Pure rotational microwave spectra have been reported by Oyama et al. [37] and McCarthy et al. [22], both of whom used an electric discharge source to produce the cis isomer. These measurements provide detailed structural information and form an excellent starting point for the present infrared experiments. In particular, by adding calculated VPT2 vibrational and isotopic shifts to the measured ground state rotational constants we obtain a reasonable predicted spectrum of the OD stretch band for fitting to our experimental spectrum.

In the cis-DOCO molecule (also a planar, near-prolate asymmetric top), the strongest OD stretch transition dipole component is aligned along the b-axis. VPT2 calculations predict that the ratio of a-type to b-type integrated band intensities for cisDOCO is $\sim 0.077$. The observed spectrum (Figure 4) is dominated by b-type transitions (propensity rule of $\Delta \mathrm{K}_{\mathrm{a}}= \pm 1$ ), signified by prominent, yet unresolved, $\mathrm{Q}$ branch transitions each originating from the ground state rotational levels of a given $\mathrm{K}_{\mathrm{a}}$ value. Here, only $\mathrm{K}_{\mathrm{a}}=1-8$ transitions are unambiguously identified. For a near-prolate asymmetric top, the frequency spacing between the $\mathrm{Q}$ branches of neighboring $\mathrm{K}_{\mathrm{a}}$ subbands is approximately given by $\mathrm{A}-(\mathrm{B}+\mathrm{C}) / 2$, or $\sim 6.6 \mathrm{~cm}^{-1}$. a-type transitions were not observed here for either carbon isotopologues of cis-DOCO.

Since b-type transitions require a change in the $\mathrm{K}_{\mathrm{a}}$ quantum number, the rotational constant A can be obtained by fitting the $\mathrm{K}_{\mathrm{a}}$ stack energy spacing. Because the ground state rotational constants for the ${ }^{12} \mathrm{C}$ isotopologue have been measured by microwave 
spectroscopy $[22,23]$, only the excited state value of $A_{v}$ and the band origin are fitted. For the ${ }^{12} \mathrm{C}$ isotopologue, the initial guess for $\mathrm{A}_{\mathrm{v}}$ is obtained from the calculated vibrational shift to the measured ground state rotational constant $\mathrm{A}_{0}$ provided by VPT 2 . For the ${ }^{13} \mathrm{C}$ isotopologue, the initial guess for $A_{v}$ is determined from the sum of the computed vibrational and isotopic shifts to the measured $\mathrm{A}_{0}$ for the ${ }^{12} \mathrm{C}$ isotopologue. For both the ground and excited state, the quartic centrifugal distortion terms are fixed to the ground state values measured by McCarthy et al. [22]. As shown in Figure 4, fitting with only the $A_{v}$ constant and band origin is sufficient to match the experimental spectrum to within experimental uncertainty. Here, the standard deviation of the fit is $0.014 \mathrm{~cm}^{-1}$. The fitted rovibrational constants for cis-DOCO are compiled in Table 3.

Comparison of the measured cis-DOCO band origin reveal nearly exact agreement $\left(\sim 1 \mathrm{~cm}^{-1}\right)$ with the values computed by MULTIMODE using the global PES by Chen et al. [29] (Table 1). The predicted band origins obtained by Guo and co-workers [38] and our MULTIMODE calculation using the PES by Wang et al. [16] (Table 2) achieved similar agreement to within $\sim 5 \mathrm{~cm}^{-1}$ of the measured value. The largest discrepancy in the predicted band origin of $\sim 15 \mathrm{~cm}^{-1}$ is observed using VPT2 (Table 3), even though the predicted vibrational and isotopic shifts to the rotational constants from VPT2 are accurate to within $\sim 100 \mathrm{MHz}$ (well below the experimental resolution) of the measured values. Finally, we note that these state-of-the-art theoretical methods accurately capture the subtle anharmonic effects that give rise to the small isotopic shifts $\left(\sim 0.2 \mathrm{~cm}^{-1}\right)$ in the vibrational transition frequencies for both DOCO isomers.

\section{Conclusion}

In this work, we report the high resolution $\left(\sim 0.03 \mathrm{~cm}^{-1}\right)$ spectroscopy of the isotopologues of DOCO isomers from the OD+CO reaction in the mid-IR (3.7 to $4.2 \mu \mathrm{m})$. Using time-resolved frequency comb spectroscopy, we have reported spectra and partial 
rovibrational analyses for the OD stretch bands of cis-DO ${ }^{12} \mathrm{CO}$, cis-DO ${ }^{13} \mathrm{CO}$, and trans$\mathrm{DO}^{13} \mathrm{CO}$. A future direction of research is the development of high power frequency comb sources in both the 5 to $7 \mu \mathrm{m}$ and 8 to $10 \mu \mathrm{m}$ wavelength regions, which cover the carbonyl $(\mathrm{C}=\mathrm{O})$ stretch and $\mathrm{D}-\mathrm{O}-\mathrm{C}$ bend mode vibrational frequencies, respectively, of both DOCO isomers. These two vibrational bands have significantly larger intensities than the OD stretch mode. Sears et al. [21] reported the only measurement of the carbonyl stretch of gas phase trans-DOCO, but this mode has not been seen for the cis isomer. These measurements at longer infrared wavelengths will provide useful spectroscopic parameters to improve the quality of the global PES used to accurately model $\mathrm{OH}(\mathrm{D})+\mathrm{CO}$ reaction kinetics and dynamics. Finally, we hope that our work will motivate further studies at even higher resolution (microwave or infrared) to complete our partial rotational analyses of these $\mathrm{H}(\mathrm{D}) \mathrm{OCO}$ radicals, ideally at much lower temperatures and pressures. 


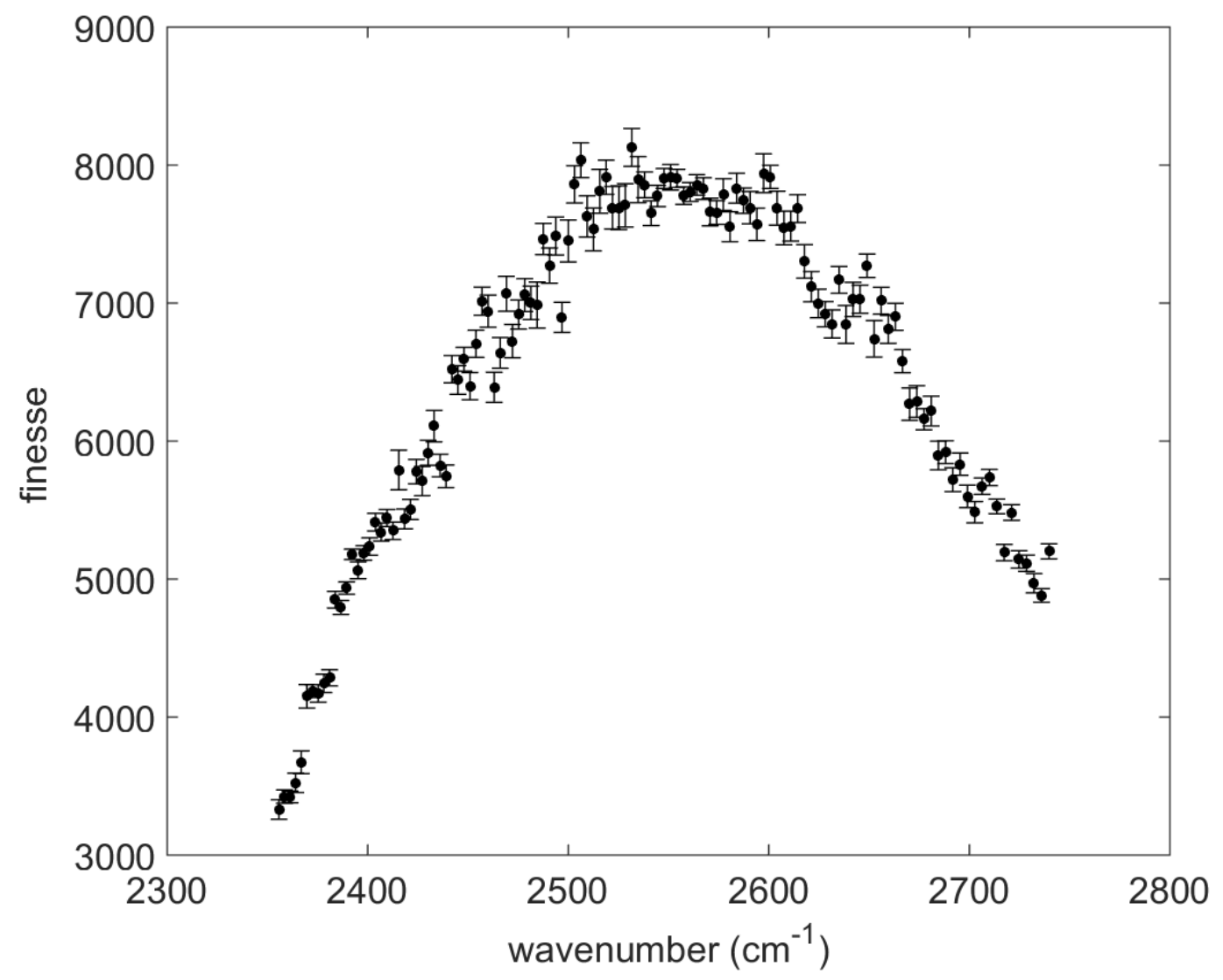

Figure 1A: Cavity finesse. The cavity finesse for a cavity length of $54.9 \mathrm{~cm}$ was obtained using the cavity ringdown technique. 


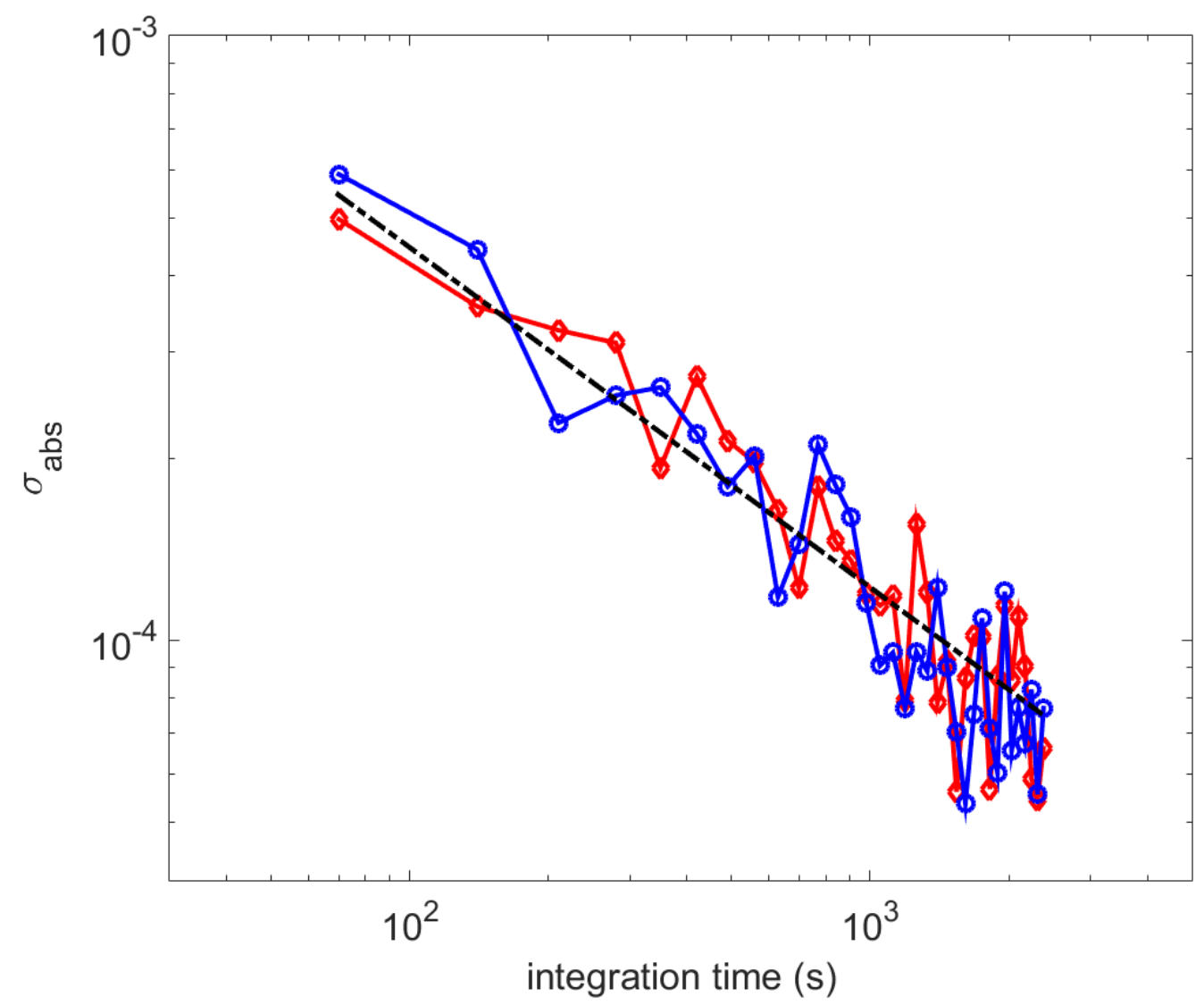

Figure 1B: Allan deviation of the absorbance determined from eq. 1. Blue and red traces correspond to the measured absorbance at two different baseline points in the spectrum. The black dashed line is the $\tau^{-1 / 2}$ dependence, where $\tau$ is the averaging time. 


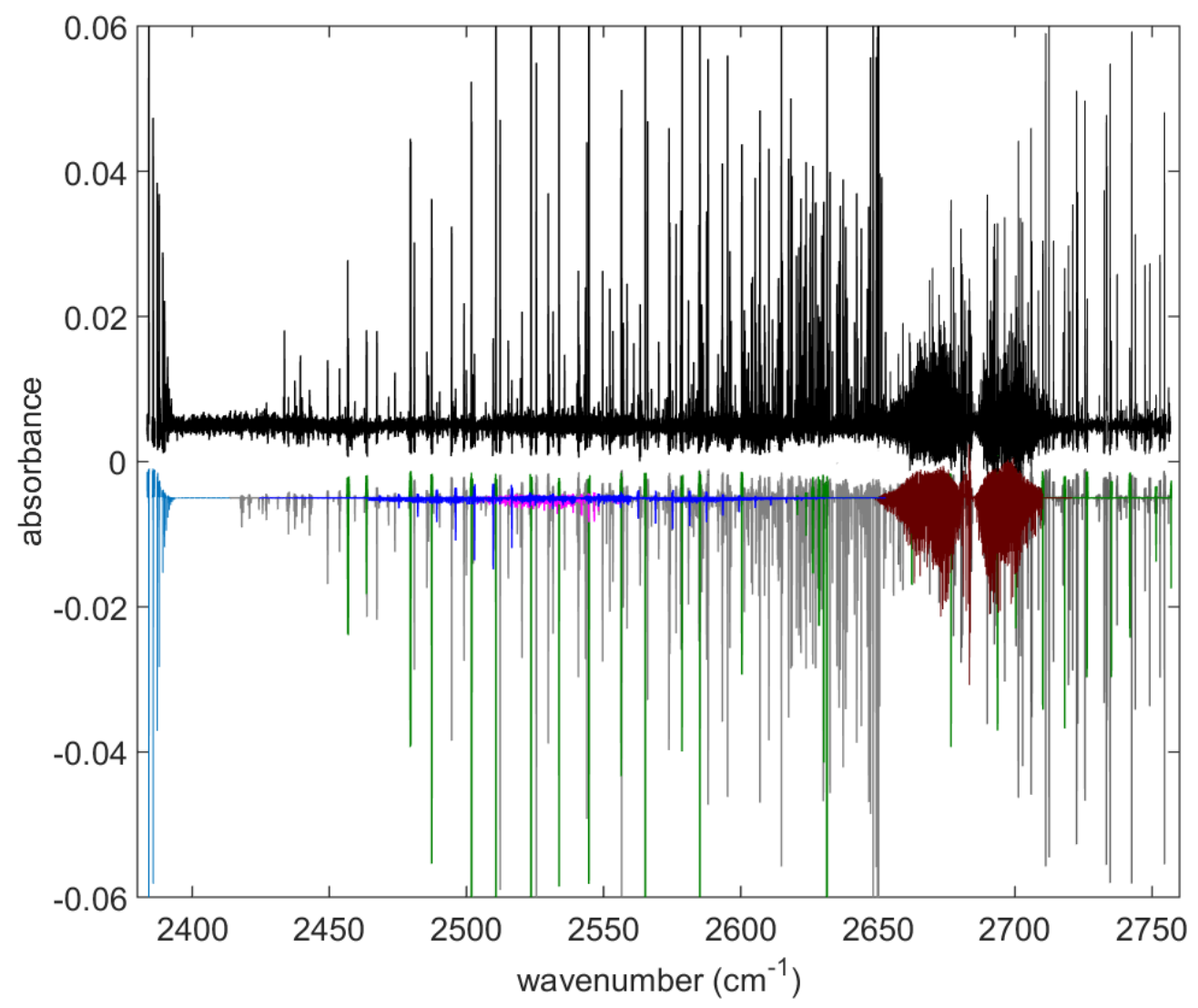

Figure 2: Spectral survey of all species from the $\mathrm{OD}+\mathrm{CO}$ reaction. $\mathrm{Cyan}$ : $\mathrm{CO}_{2}$; blue: cis-DO ${ }^{12} \mathrm{CO}$; brown: trans-DO ${ }^{12} \mathrm{CO}$; pink: $\mathrm{DO}_{2}$; green: OD; gray: $\mathrm{D}_{2} \mathrm{O}$. 


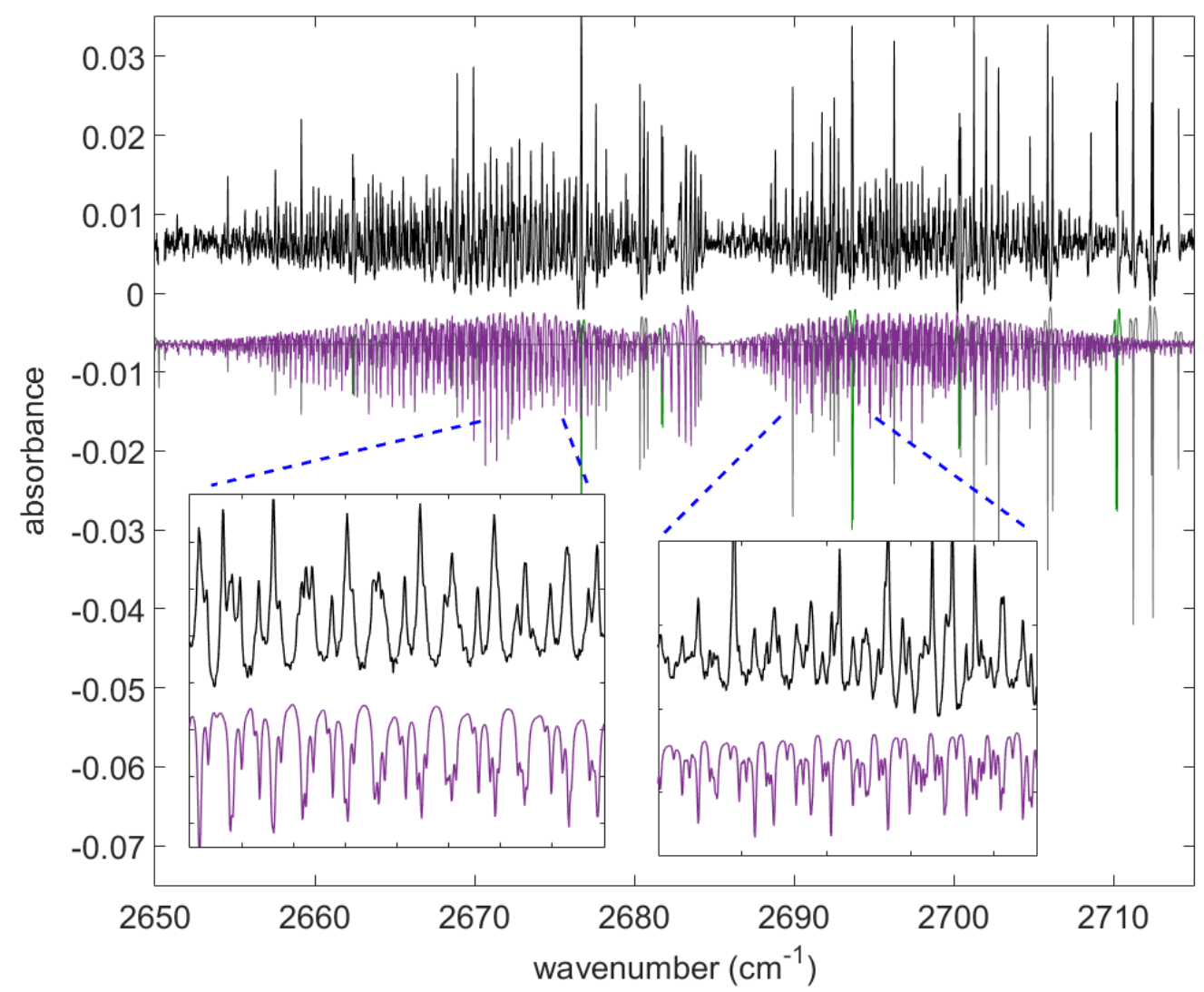

Figure 3: Experimental trans-DO ${ }^{13} \mathrm{CO}$ spectrum and fit (inverted). In the insets, the simulated OD (green) and $\mathrm{D}_{2} \mathrm{O}$ (gray) lines have been removed for clarity. 


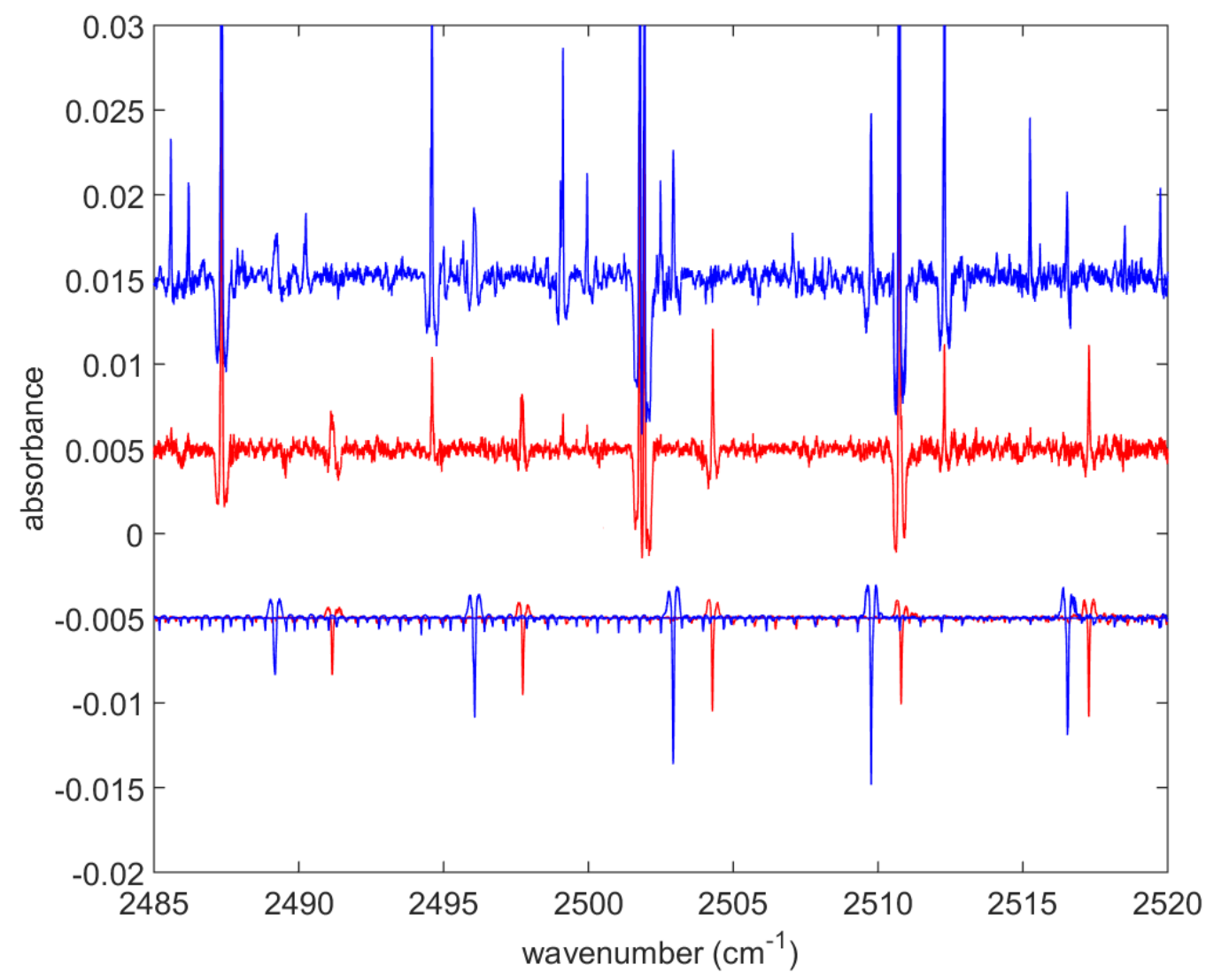

Figure 4: Carbon isotopologues of cis-DOCO. blue: $c$ is-DO ${ }^{12} \mathrm{CO}$; red: $c$ is-DO ${ }^{13} \mathrm{CO}$.

The simulated spectra for cis-DOCO are shown inverted. In the inverted spectra, the simulated $\mathrm{OD}$ and $\mathrm{D}_{2} \mathrm{O}$ lines have been excluded for clarity. 
Table 1: Vibrational frequencies using MULTIMODE

\begin{tabular}{lrrrr} 
Mode & $c i s-\mathrm{DO}^{12} \mathrm{CO}^{\mathrm{c}}$ & cis-DO & ${ }^{13} \mathrm{CO}^{\mathrm{c}}$ & trans-DO \\
& 451.57 & 447.26 & 396.72 & 393.92 \\
\hline 1 torsion & 535.12 & 530.95 & 589.11 & 582.64 \\
2 O-C-O bend & 957.22 & 955.59 & 902.55 & 900.86 \\
3 H-O-C bend & 1116.05 & 1091.19 & 1083.79 & 1063.13 \\
4 C-O stretch & 1818.27 & 1777.48 & 1851.55 & 1813.78 \\
5 C=O stretch & 2540.93 & 2540.77 & 2686.2 & 2686.25 \\
6 O-D stretch & & & & \\
\hline
\end{tabular}

cPES from Chen et al. [29]

\begin{tabular}{|c|c|c|c|c|}
\hline Mode & cis- $-\mathrm{DO}^{12} \mathrm{CO}^{\mathrm{d}}$ & $c i s-\mathrm{DO}^{13} \mathrm{CO}^{\mathrm{d}}$ & trans $-\mathrm{DO}^{12} \mathrm{CO}^{\mathrm{d}}$ & trans $-\mathrm{DO}^{13} \mathrm{CO}^{\mathrm{d}}$ \\
\hline 1 torsion & 460.51 & 455.95 & 392.35 & 389.59 \\
\hline $2 \mathrm{O}-\mathrm{C}-\mathrm{O}$ bend & 536.97 & 532.73 & 587.86 & 581.36 \\
\hline $3 \mathrm{H}-\mathrm{O}-\mathrm{C}$ bend & 953.91 & 952.69 & 900.55 & 898.95 \\
\hline $4 \mathrm{C}-\mathrm{O}$ stretch & 1118.85 & 1093.81 & 1083.95 & 1063.15 \\
\hline $5 \mathrm{C}=\mathrm{O}$ stretch & 1820.93 & 1780.43 & 1852.83 & 1814.09 \\
\hline 6 O-D stretch & 2544.57 & 2544.4 & 2685.54 & 2685.91 \\
\hline
\end{tabular}

${ }^{\mathrm{d}} \mathrm{PES}$ from Wang et al. [16]

Table 2: Vibrational frequencies using CCSD(T)/ANO1 VPT2 (in cm-1)

\begin{tabular}{lrrrr} 
Mode & cis-DO ${ }^{12} \mathrm{CO}$ & cis-DO ${ }^{13} \mathrm{CO}$ & trans-DO ${ }^{12} \mathrm{CO}$ & trans-DO ${ }^{13} \mathrm{CO}$ \\
\hline 1 torsion & 453.6757 & 449.1907 & 394.5547 & 392.2696 \\
2 O-C-O bend & 535.0366 & 530.9112 & 587.5528 & 581.4452 \\
3 H-O-C bend & 963.4104 & 958.6848 & 899.2502 & 898.1702 \\
4 C-O stretch & 1121.2705 & 1095.9654 & 1080.4844 & 1059.739 \\
5 C=O stretch & 1814.7033 & 1773.6179 & 1847.3807 & 1783.3468 \\
6 O-D stretch & 2555.4755 & 2555.2693 & 2688.1609 & 2688.3992 \\
\hline
\end{tabular}


Table 3: trans-DOCO and cis-DOCO molecular constants (in MHz)

\begin{tabular}{lrrrr} 
Parameter & trans $-\mathrm{DO}^{12} \mathrm{CO}$ & trans $-\mathrm{DO}^{13} \mathrm{CO}$ & cis-DO ${ }^{12} \mathrm{CO}$ & cis-DO ${ }^{13} \mathrm{CO}$ \\
\hline$v_{0}\left(\mathrm{~cm}^{-1}\right)$ & $2684.1^{\mathrm{a}}$ & $2684.159(2)$ & $2539.909(3)$ & $2539.725(4)$ \\
$\mathrm{A}_{0}$ & $154685.537^{\mathrm{b}}$ & 148175.6034 & $110105.52^{\mathrm{b}}$ & $106124(5)$ \\
$\mathrm{B}_{0}$ & $10685.952^{\mathrm{b}}$ & --- & $11423.441^{\mathrm{b}}$ & 11420.075 \\
$\mathrm{C}_{0}$ & $9981.624^{\mathrm{b}}$ & --- & $10331.423^{\mathrm{b}}$ & 10291.999 \\
$\left(\mathrm{~B}_{0}+\mathrm{C}_{0}\right) / 2$ & $10333.788^{\mathrm{b}}$ & $10310(1)$ & $10877.432^{\mathrm{b}}$ & 10856.037 \\
$\mathrm{~A}_{\mathrm{v}}$ & $153431.4^{\mathrm{a}}$ & 147030.0607 & $109313(4)$ & $105423(5)$ \\
$\mathrm{B}_{\mathrm{v}}$ & $10671.06^{\mathrm{a}}$ & --- & 11422.882 & 11419.559 \\
$\mathrm{C}_{\mathrm{v}}$ & $9963.386^{\mathrm{a}}$ & --- & 10324.228 & 10284.951 \\
$\left(\mathrm{~B}_{\mathrm{v}}+\mathrm{C}_{\mathrm{v}}\right) / 2$ & $10317.223^{\mathrm{a}}$ & $10293(1)$ & 10873.555 & 10852.255 \\
\hline
\end{tabular}

a Petty and Moore [18]

b. McCarthy et al. [22]

\section{References}

[1]D. Herschbach, Faraday Discussions 142, 9 (2009).

[2]G. Porter, In The Chemical Bond: Structure and Dynamics, edited by Ahmed H. Zewail (Academic Press, Boston, 1992), p. 113-148.

[3]J.L. Hall and T.W. Hänsch, in Femtosecond Optical Frequency Comb Technology: Principle, Operation and Application, edited by J. Ye and S.T. Cundiff (Springer Science Business Media, New York) pp. 1-11.

[4]A. Marian, M. C. Stowe, J. R. Lawall, D. Felinto, and J. Ye, Science 306 (5704), 2063 (2004); M. J. Thorpe, K. D. Moll, R. J. Jones, B. Safdi, and J. Ye, Science 311 (5767), 1595 (2006).

[5]P.B. Changala, B. Spaun, D. Patterson, J.M. Doyle, and J. Ye, Applied Physics B 122 (12), 292 (2016).

[6]B. Spaun, P. B. Changala, D. Patterson, B. J. Bjork, O. H. Heckl, J. M. Doyle, and J. Ye, Nature 533 (7604), 517 (2016).

[7]B. J. Bjork, T. Q. Bui, O. H. Heckl, P. B. Changala, B. Spaun, P. Heu, D. Follman, C. Deutsch, G. D. Cole, M. Aspelmeyer, M. Okumura, and J. Ye, Science 354 (6311), 444 (2016).

[8]T.Q. Bui, B.J. Bjork, P.B. Changala, H. Heckl, B. Spaun, and J. Ye, Chemical Physics Letters 683, 91 (2017).

[9]T.Q. Bui, B.J. Bjork, P.B. Changala, T.L. Nguyen, J.F. Stanton, M. Okumura, and J. Ye, Science Advances 4 (1) (2018).

[10]A. J. Fleisher, B. J. Bjork, T. Q. Bui, K. C. Cossel, M. Okumura, and J. Ye, Journal of Physical Chemistry Letters 5 (13), 2241 (2014).

[11]K. Iwakuni, G. Porat, T.Q. Bui, B.J. Bjork, S.B. Schoun, O.H. Heckl, M.E. Fermann, and J. Ye, ArXiv e-prints 1803 (2018). <http://adsabs.harvard.edu/abs/2018arXiv180300623I>.

[12]J. S. Francisco, J. T. Muckerman, and H. G. Yu, Accounts of Chemical Research 43 (12), 1519 (2010); C. J. Johnson, R. Otto, and R. E. Continetti, Physical Chemistry Chemical Physics 16 (36), 19091 (2014). 
[13]C. J. Johnson and R. E. Continetti, Journal of Physical Chemistry Letters 1 (12), 1895 (2010); H. Guo, International Reviews in Physical Chemistry 31 (1), 1 (2012).

[14]J. Y. Ma, J. Li, and H. Guo, Journal of Physical Chemistry Letters 3 (17), 2482 (2012); X. C. Huang, R. C. Fortenberry, Y. M. Wang, J. S. Francisco, T. D. Crawford, J. M. Bowman, and T. J. Lee, Journal of Physical Chemistry A 117 (32), 6932 (2013).

[15]J. Li, Y. M. Wang, B. Jiang, J. Y. Ma, R. Dawes, D. Q. Xie, J. M. Bowman, and H. Guo, Journal of Chemical Physics 136 (4), 041103 (2012).

[16]Y. M. Wang, S. Carter, and J. M. Bowman, Journal of Physical Chemistry A 117 (39), 9343 (2013).

[17]T. L. Nguyen, B. C. Xue, R. E. Weston, J. R. Barker, and J. F. Stanton, Journal of Physical Chemistry Letters 3 (11), 1549 (2012); R. E. Weston, T. L. Nguyen, J. F. Stanton, and J. R. Barker, Journal of Physical Chemistry A 117 (5), 821 (2013); X. H. Wang and J. M. Bowman, Journal of Physical Chemistry A 118 (4), 684 (2014).

[18]J. T. Petty and C. B. Moore, Journal of Chemical Physics 99 (1), 47 (1993).

[19]J. T. Petty and C. B. Moore, Journal of Molecular Spectroscopy 161 (1), 149 (1993).

[20]C. H. Chang, G. T. Buckingham, and D. J. Nesbitt, Journal of Physical Chemistry A 117 (50), 13255 (2013).

[21]T. J. Sears, W. M. Fawzy, and P. M. Johnson, Journal of Chemical Physics 97 (6), 3996 (1992).

[22]M. C. McCarthy, O. Martinez, B. A. McGuire, K. N. Crabtree, M. A. MartinDrumel, and J. F. Stanton, Journal of Chemical Physics 144 (12), 11, 124304 (2016).

[23]T. Oyama, W. Funato, Y. Sumiyoshi, and Y. Endo, Journal of Chemical Physics 134 (17), 7, 174303 (2011).

[24]H. E. Radford, W. Wei, and T. J. Sears, Journal of Chemical Physics 97 (6), 3989 (1992); H. E. Radford, M. A. Moore, T. J. Sears, J. Grussdorf, J. Nolte, and F. Temps, Journal of Molecular Spectroscopy 165 (1), 137 (1994).

[25]F. Adler, K. C. Cossel, M. J. Thorpe, I. Hartl, M. E. Fermann, and J. Ye, Optics Letters 34 (9), 1330 (2009).

[26]F. Adler, M. J. Thorpe, K. C. Cossel, and J. Ye, in Annual Review of Analytical Chemistry, Vol 3, edited by E. S. Yeung and R. N. Zare (Annual Reviews, Palo Alto, 2010), Vol. 3 pp. 175-205.

[27]L. Nugent-Glandorf, T. Neely, F. Adler, A. J. Fleisher, K. C. Cossel, B. Bjork, T. Dinneen, J. Ye, and S. A. Diddams, Optics Letters 37 (15), 3285 (2012).

[28]J. M. Bowman, S. Carter, and X. C. Huang, International Reviews in Physical Chemistry 22 (3), 533 (2003).

[29]J. Chen, X. Xu, X. Xu, and D. H. Zhang, Journal of Chemical Physics 138 (22), 4, 221104 (2013).

[30]I.M. Mills, in Molecular Spectroscopy: Modern Research (Academic Press, 1972) pp. 115-140.

[31]L. McCaslin and J. Stanton, Molecular Physics 111 (9-11), 1492 (2013).

[32]J.F. Stanton, Gauss, J., Cheng, J., Harding, M.E., Matthews, D.A., Szalay, P.G., with contributions from Auer, A.A., Bartlett, R.J., Benedikt, U., Berger, C., Bernholdt, D.E., Bomble, Y.J.,Christiansen, O., Engel, F., Faber, R., Heckert, M., Heun, O., Hilgenberg, M., Huber, C., Jagau, T.-C., Jonsson, D., Jusélius, J., Kirsch, T., Klein, K., Lauderdale, W.J., Lipparini, F., Metzroth T., Mück, L.A., 
O'Neill, D.P., Price, D.R., Prochnow, E., Puzzarini, C., Ruud, K.,Schiffmann, F., Schwalbach, W., Simmons, C., Stopkowicz, S., Tajti, A., Vázquez, J., Wang, F., Watts, J.D. and the integral packages MOLECULE (Almlöf, J. and Taylor, P.R.), PROPS (Taylor, P.R.), ABACUS (Helgaker, T., Jensen, H.J.Aa., Jørgensen, H.P., and Olsen, J.), and ECP routines by Mitin, A.V. and Wüllen C. van, CFOUR, Coupled-Cluster techniques for Computational Chemistry, a quantum-chemical program package (2018).

[33]M. C. Abrams, S. P. Davis, M. L. P. Rao, and R. Engleman, Journal of Molecular Spectroscopy 165 (1), 57 (1994).

[34]R. A. Toth, Journal of Molecular Spectroscopy 195 (1), 98 (1999).

[35]K. G. Lubic, T. Amano, H. Uehara, K. Kawaguchi, and E. Hirota, Journal of Chemical Physics 81 (11), 4826 (1984).

[36]C. M. Western, Journal of Quantitative Spectroscopy \& Radiative Transfer 186, 221 (2017).

[37]T. Oyama, Y. Sumiyoshi, and Y. Endo, Journal of Chemical Physics 137 (15), 6, 154307 (2012).

[38]J. Wang, J. Li, J. Y. Ma, and H. Guo, Journal of Chemical Physics 140 (18), 7, 184314 (2014).

\section{Acknowledgements}

We thank H. Guo for stimulating discussions. We acknowledge financial support from AFOSR, DARPA SCOUT, NIST, and NSF JILA Physics Frontier Center. J.F. Stanton acknowledges financial support from the U.S. Department of Energy, Office of Basic Energy Sciences for Award DE-FG02-07ER15884. J. M. Bowman thanks the National Science Foundation (CHE-1463552) for financial support. T. Q. Bui is supported by the National Research Council Research Associate Fellowship, P. B. Changala is supported by the NSF GRFP. 